\title{
ASYMPTOTIC GIANT BRANCH STARS: THERMAL PULSES, CARBON PRODUCTION, AND DREDGE UP; NEUTRON SOURCES AND S-PROCESS NUCLEOSYNTHESIS
}

\author{
Icko Iben, Jr. \\ University of Illinois \\ Department of Astronomy \\ 1002 West Green Street \\ Urbana, Illinois 61801 , USA
}

ABSTRACT. A brief review is given of the structure of asymptotic giant branch (AGB) stars and of the characteristics of the thermal pulses which these stars experience. Following a pulse, model AGB stars with a large core mass easily dredge up fresh carbon, which is the main product of incomplete helium burning, and s-process isotopes, which are made as a consequence of the activation of the ${ }^{22} \mathrm{Ne}$ neutron source. Model AGB stars of small core mass activate the ${ }^{13} C$ neutron source and produce s-process isotopes in nearly the solar system distribution. They also dredge up fresh carbon and s-process isotopes, but only if overshoot or some other form of "extra" mixing beyond the lower boundary of the convective envelope is invoked.

\section{PROGENITOR MASS, CORE MASS \& COMPOSITION, AND LOCATION IN THE HR DIAGRAM}

Every star massive enough to evolve off the main sequence in less than the Hubble time $\left(10-15 \times 10^{9} \mathrm{yr}\right)$ and less massive than about $8 M_{\odot}$ evolves into an asymptotic giant branch star with an electron-degenerate core composed of carbon and oxygen. The ascent of the AGB begins following the exhaustion of helium at the center. During the early AGB (EAGB) phase, the electron-degenerate helium-exhausted, or carbon-oxygen $(\mathrm{CO})$ core grows until its outer edge comes very close (in mass) to the base of the hydrogen-rich envelope. Thereafter, helium and hydrogen burn alternately in shells and the star is said to be in the thermally pulsing AGB (TPAGB) phase.

Model AGB stars are confined to a very small region of the theoretical H$R$ diagram, all with surface temperatures in the range 2500-6000 K. During the EAGB phase, those of very small initial $\mathrm{CO}$ core mass (total initial mass less than $M \sim 2 M_{\odot}$ ) follow a path very close to the red giant branch defined by shell hydrogen-burning stars with electron-degenerate helium cores (hence the designation "asymptotic"). When they begin to thermally pulse with $\mathrm{CO}$ cores of mass $M_{C O} \sim 0.5 M_{\odot}$, such "low mass" AGB stars are brighter than the tip of the red 
giant branch $(\log L \sim 3.3)$. Models which have more massive $\mathrm{CO}$ cores at the start of the TPAGB phase ("high mass" AGB stars) have luminosities which are correspondingly larger. The most massive initial $\mathrm{CO}$ core mass which can be formed is $1.1 M_{\odot}$.

Stars more massive than about $8 M_{\odot}$ and less massive than about $10 M_{\odot}$ do not develop electron-degenerate cores until after the exhaustion of central carbon. These stars also experience thermal pulses (Nomoto 1984), but the full character of these pulses has not yet been explored. At the onset of the TPAGB phase in these "very high mass" AGB stars, the mass of the carbon-exhausted, or oxygen-neon (ONe) core varies from $1.1 M_{\odot}$ to $\sim 1.38 M_{\odot}$, depending on the mass of the initial main sequence progenitor.

Stars more massive than $\sim 10 M_{\odot}$ become supernovae after the electrondegenerate core of iron peak elements which they form collapses to become a neutron star or black hole.

\section{FINAL FATE - ENVELOPE EJECTION; PLANETARY NEBULA AND WHITE DWARF PHASES}

If the mass of the $\mathrm{CO}$ core in a high mass $\mathrm{AGB}$ star were to reach the effective Chandrasekhar limit of about $1.4 M_{\odot}$, this core would explode, liberating energy of supernova proportions and leaving no remnant. If we assume a birthrate proportional to the Salpeter mass function and adopt a total stellar deathrate in the Galaxy of $1 y^{-1}$, it follows that, if all stars of initial mass in the range $1.4-8 M_{\odot}$ were to continue as AGB stars until their core masses reached the effective Chandrasekhar limit, the supernovae rate in our Galaxy would be some 20-40 times larger than the observed rate (van den Bergh, McClure, \& Evans 1987; Evans, van den Bergh, \& McClure 1989). If this were true of stars in the $8-10 M_{\odot}$ range as well, the discrepancy would be exacerbated. We must conclude that the vast majority of AGB stars lose their hydrogen-rich envelopes before their core masses grow to the Chandrasekhar limit.

It is known that red giants of all kinds lose mass at a fairly high rate, as summarized, for example, in the Reimers (1975) analysis. However, even with the Reimers rate, enough AGB stars (those with the largest initial masses) would survive until their core masses reached the Chandrasekhar limit that the predicted supernova rate would still exceed the observed supernova rate. Hence, real AGB stars of high mass must at some point lose mass more rapidly than the Reimers rate. The simplest interpretation is that, depending on its core mass and envelope mass, an AGB star encounters a secular instability that forces its hydrogen-rich envelope to be ejected very rapidly. Tuchman, Sack, and Barkat $(1978,1979)$ have identified a mechanism which may be responsible for this high rate, but there are many other candidates (e.g., Bowen 1989). OH-IR stars, some of which are known to lose mass as rapidly as $10^{-4} M_{\odot} y r^{-1}$ (e.g., Knapp \& Morris 1985, Jura 
1989), are probably descendants of initially massive AGB stars which are in the process of ejecting their envelopes. Once envelope ejection is completed and the compact remnant (the original core of the AGB star) contracts to achieve surface temperatures greater than $\sim 30,000 K$, these stars will appear as planetary nebulae for a brief time due to the excitation of atoms in the ejected nebula by photons emitted by the compact remnant, which will eventually cool down to become a white dwarf. The globular cluster M15 contains a planetary nebula, and this tells us that even stars initially as light as $0.8 M_{\odot}$ eject their envelopes rapidly.

From the observed properties of AGB stars in clusters in the Magellanic clouds (Frogel, Mould, \& Blanco 1990), coupled with a theoretical relationship between luminosity and core mass, one can ascertain that initially low mass stars $\left(M \leq 2 M_{\odot}\right)$ lose their hydrogen-rich envelope before having added more than about $0.1 M_{\odot}$ to the CO core they possessed at the beginning of the TPAGB phase (namely, $\sim 0.5 M_{\odot}$ ), whereas initially more massive stars do not increase their core mass by more than a few times $0.01 M_{\odot}$ during the TPAGB phase before ejecting their envelope. Thus, a combination of theoretical and observational data tells us that stars of initial mass in the $1-2 M_{\odot}$ range produce $\mathrm{CO}$ white dwarfs of mass about $0.6 M_{\odot}$, stars of initial mass in the $2-8 M_{\odot}$ range produce $\mathrm{CO}$ white dwarfs in the $0.6-1.1 M_{\odot}$ range, and most of the stars of initial mass in the $8-10 M_{\odot}$ range produce $\mathrm{ONe}$ white dwarfs in the $1.1-1.38 M_{\odot}$ range. Only those stars with initial masses near $10 M_{\odot}$ will grow ONe cores as large as the effective Chandrasekhar limit of $1.38 M_{\odot}$. On reaching the Chandrashekhar mass the core begins to collapse due to electron capture on a trace of ${ }^{24} \mathrm{Mg}$ present in the core (Nomoto 1984, 1987; Miyaji \& Nomoto 1987). The hydrogen-rich envelope is ejected and the core evolves into a neutron star.

\section{BASIC STRUCTURE - CORE, HALO, AND "NUCLEAR-ACTIVE" ZONE}

The core of an AGB star is essentially a hot white dwarf and hence has a radius of $\sim 10^{-2} R_{\odot}$; during the TPAGB phase, its mass is in the range $0.5-1.38 M_{\odot}$; the larger the initial mass of the star, the more massive the core. Most of the hydrogen-rich material in the star is spread out over a vast volume of radial size in the range $\sim 100-1000 R_{\odot}$. Between the core and the envelope is a small region in which both helium- and hydrogen-burning take place in alternation. This region may be called the "nuclear-active" zone. Most of the time, hydrogen is burning quiescently above two inert layers, the lower one containing helium and products of partial helium burning, the upper one containing mostly helium plus the main product of CNO cycling, ${ }^{14} \mathrm{~N}$. The hydrogen-burning shell, which is located only a core radius or so above the edge of the core and which deposits helium and nitrogen onto the inert layer immediately below it, has a mass which varies from $\sim 10^{-4} M_{\odot}$ when $M_{C O} \sim 0.6 M_{\odot}$ to $\sim 2 \times 10^{-7} M_{\odot}$ when $M_{O N e} \sim 1.36 M_{\odot}$. And, yet, the luminosity during the quiescent hydrogen-burning phase ranges from $\sim 6 \times 10^{3} L_{\odot}$ to $\sim 5 \times 10^{4} L_{\odot}$ over the same core mass range, so burning rates per unit mass are 
quite intense.

The core is hot for two reasons: (1) heat is continuously being released by matter being compressed in the outer edge of the core in response to pressure from the growing inert layer above it, and (2) this heat is carried inward efficiently by electron conduction. Temperatures in the core do not rise indefinitely since a hot, degenerate electron plasma loses energy by the emission of neutrinos; the balance between heating and cooling maintains the mean temperature in the core at about $10^{8} \mathrm{~K}$. Electron degeneracy is quite high through most of the core, as central densities vary from $\sim 10^{6} \mathrm{~g} \mathrm{~cm}^{-3}$ to $\sim 10^{9} \mathrm{~g} \mathrm{~cm}^{-3}$ and densities at the core edge are of the order of $10^{5} \mathrm{~g} \mathrm{~cm}^{-3}$.

That the envelope of the star forms an extended halo around the core is a general property of hot, high entropy matter which is placed around a massive point source (Fujimoto \& Iben 1990). The high temperatures at the base of the envelope (and so the high entropy of the envelope) are a consequence of the existence of a nuclear-active zone between the core and the envelope. The large entropy gradient in the burning shell has the consequence of forcing the density of matter above the shell to fall off more steeply than the inverse square of the radius over a distance which is very large compared with the radius of the core (but small, of course, relative to the size of the envelope).

\section{THERMAL PULSES (= HELIUM SHELL FLASHES)}

During the quiescent hydrogen-burning phase, the temperatures and densities in the helium-rich layers below the burning shell increase as the mass of these layers increases. Once the mass of the helium-rich region exceeds a critical value, the rate at which energy is emitted by helium burning becomes larger than the rate at which this energy can escape via radiative diffusion, and a thermonuclear runaway ensues.

Insight into the cause of the runaway comes from model studies of accreting white dwarfs. Whatever the accreted fuel, this fuel can burn stably and as rapidly as it is accreted for accretion rates lying between two limits that differ by a factor of $\sim 2-3$ (the precise value of the factor depends on the mass of the white dwarf). If the accretion rate is smaller than the lower limit, nuclear burning is unstable; $a$ flash occurs whenever the amount of accumulated fuel exceeds a critical value which is smaller, the larger the mass of the white dwarf. Between flashes, the accretion layer is inert. If the accretion rate is larger than the upper limit, the fuel burns stably at a rate equal to the upper limit; now, however, since fuel is not burned as rapidly as it is accumulated, the layer of unburned fuel continues to increase in mass and expands to red giant dimensions. The two limits define a band in the accretion rate - white dwarf mass plane.

If the accreted material is helium, the accretion rate at the center of the band varies from $\sim 4 \times 10^{-7} M_{\odot} y r^{-1}$, for a white dwarf mass of $\sim 0.6 M_{\odot}$, to $\sim 4 \times$ 
$10^{-6} M_{\odot} y r^{-1}$, for a white dwarf mass of $\sim 1.1 M_{\odot}$ or larger (Iben \& Tutukov 1989). If the accreted material is hydrogen, the accretion rate at the center of the stable band is $\sim$ ten times smaller, for any given white dwarf mass, than is the accretion rate at the center of the stable band for helium accretion.

During the quiescent hydrogen-burning phase, the hydrogen-rich portion of an AGB star is analogous to the configuration formed by a white dwarf accreting hydrogen more rapidly than it can burn hydrogen. However, since the hydrogenburning shell is adding helium to a helium layer above a white dwarf, the hydrogenexhausted portion of the AGB star is analogous to a white dwarf accreting helium at a rate which is about ten times smaller than the rate at which helium can burn stably. Of necessity, the helium layer must periodically experience a thermonuclear runaway.

The thermonuclear runaway is initiated at the base of the lower helium-rich layer. For a core mass of $0.6 M_{\odot}$, the amount of freshly accreted matter required to achieve ignition is about $0.01 M_{\odot}$ and, for $M_{C O} \sim 1.0 M_{\odot}$, it is about $0.0008 M_{\odot}$. The high burning rates achieved cause the formation of a convective layer which extends from the base of the helium-burning region almost to the base of the hydrogenburning shell. Along with the rapid increase in the thermal energy of matter in and around the burning zone, there is a rapid increase in pressures, and this forces matter in and above the burning region to expand outward. Most of the energy flowing outward from the burning region goes into increasing the store of thermal energy in the convective shell and into raising the potential energy of the outwardly expanding matter. Matter within and near the hydrogen-burning shell is propelled outward and cools to such an extent that hydrogen-burning is extinquished, and this leads to a temporary drop in the stellar luminosity. In response to the drop in the flux of energy passing through it, the outer portion of the hydrogen-rich envelope contracts and the surface luminosity increases until it actually exceeds the luminosity just prior to the onset of the thermal pulse.

The rate of release of nuclear energy in the helium-burning region reaches a peak (typically $10^{7}-10^{8} L_{\odot}$ ) and, in consequence of expansion, temperatures in the burning zone begin to decline, bringing about an initially rapid decrease in the rate of nuclear energy release. Convection in a shell ceases and the star settles into a quiescent helium-burning phase during which the surface luminosity is due almost entirely to the release of helium-burning energy. The surface luminosity decreases slowly to be about a factor of $1.5\left(M_{C O} \sim 1.0 M_{\odot}\right)-2.5\left(M_{C O} \sim 0.6 M_{\odot}\right)$ dimmer than during the preceding quiescent hydrogen-burning phase. Eventually, when almost as much helium has been burned as was processed by the hydrogenburning shell following the immediately preceding thermal pulse, hydrogen burning is reignited and helium burning ceases; the star now embarks on another long phase of quiescent hydrogen burning.

The phase of intense helium burning in a convective shell lasts until approximately $20 \%$ of the matter in the shell is ${ }^{12} \mathrm{C}$. Early on in this phase, all of the ${ }^{14} \mathrm{~N}$ 
which has been inherited from the hydrogen-burning shell is converted into ${ }^{22} \mathrm{Ne}$. In AGB stars with a large core mass, temperatures become large enough that a substantial fraction of this ${ }^{22} \mathrm{Ne}$ has been converted into ${ }^{25} \mathrm{Mg}$ with the release of a neutron. In AGB stars of small core mass, only 1\% of the ${ }^{22} \mathrm{Ne}$ is burned, and the major source of neutrons is the ${ }^{13} C(\alpha, n){ }^{16} O$ reaction.

The nature of the neutron-capture nucleosynthesis that occurs in the two cases is quite different. In stars with low mass cores which activate the ${ }^{13} \mathrm{C}$ neutron source, the distribution of s-process isotopes appearing in the final product is close to the solar system distribution. In stars with massive cores which activate the ${ }^{22} \mathrm{Ne}$ source, the final distribution of abundances resembles the solar system distribution in gross outline, but there are significant departures from the solar system distribution near critical branching points where abundances are particularly sensitive to the temperature and to the neutron density during the neutron-irradiation phase.

\section{NUCLEOSYNTHESIS AND DREDGE UP IN AGB STARS WITH A LARGE CORE MASS}

\subsection{Global Characteristics Versus Core Mass}

The luminosity during the quiescent hydrogen-burning phase depends on the choice of $l / H$, the ratio of mixing length to scale height in the algorithm normally used to estimate the temperature gradient in convective regions. For any given core mass, as $l / H$ is increased above a critical value, which depends on core mass and total mass, the surface luminosity increases as a larger and larger fraction of the hydrogenburning shell appears within and near the base of the convective envelope (e.g., Iben 1975a, 1976; Blöcker \& Schönberner 1990). If $l / H$ is chosen below this limit, there exists a core mass - luminosity relationship given approximately by $L_{\max } \sim$ $(5.925+0.415 x)\left(M_{C O}-0.495+0.0505 x\right) \times 10^{4} L_{\odot}$, where $x=\left[\left(M-M_{C O}\right) / 6.04\right]^{1.83}$, and masses are in solar units (Iben 1981); this relationship has been designed to fit AGB models with cores of both small and large mass and it is strictly valid only immediately prior to the onset of a pulse.

The time between pulses is a very strong function of the mass of the core, and may be approximated by $\tau_{\text {interpulse }} \sim 1300 y r\left(M_{C O} / M_{\odot}\right)^{10}$. The strength of a pulse may be measured by the peak helium-burning luminosity. This strength builds up with each succesive pulse until a "locally" asymptotic value is reached. For a core mass of $\sim 1 M_{\odot}$ the peak value is typically $\sim 10^{7} L_{\odot}$. The duration of the convective shell phase during a thermal pulse decreases steadily with increasing core mass, varying from $\sim 13 \mathrm{yr}$ when $M_{C O} \sim 0.96 M_{\odot}$ to $\sim 5$ months when $M_{O N e} \sim 1.36 M_{\odot}$. Maximum temperatures at the base of the convective shell at the asymptotic limit vary from $\sim 310 \times 10^{6} \mathrm{~K}$ to $\sim 415 \times 10^{6} \mathrm{~K}$ over the same range in core masses (Iben 1977). 


\subsection{Helium-Burning Reactions in the Convective Shell}

The basic reaction that triggers a thermonuclear runaway is the "triple alpha" reaction in which two alpha particles form an unstable beryllium nucleus which lives long enough to capture another alpha particle and form a compound nucleus with an excitation energy that is (remarkably) very close to the that of ${ }^{12} C^{* *}$ $\left({ }^{12} C\right.$ in its second excited state). This means that one can estimate the rate of the reaction simply by determining the abundance of ${ }^{12} C^{* *}$ in thermal equilibrium with ${ }^{4} \mathrm{He}$ and multiplying by the radiative lifetime of ${ }^{12} \mathrm{C}^{* *}$. In contrast, the rate of the following reaction, ${ }^{12} C(\alpha, \gamma){ }^{16} O$, is highly uncertain. Fortunately, the convective shell lasts only long enough for the ${ }^{12} C$ abundance to reach a value of about $20 \%$ by mass, and by this time the amount of ${ }^{16} \mathrm{O}$ that has been built up is much smaller than the abundance of ${ }^{12} C$, regardless of the rate of the ${ }^{12} C(\alpha, \gamma){ }^{16} O$ reaction (within the uncertainties). Thus, matter which has been processed in the outer portion of a convective shell and which eventually reaches the surface has experienced only partial helium burning and the overwhelmingly dominant nuclear species that originates in a convective shell and eventually makes its way to the surface is ${ }^{12} \mathrm{C}$. Of course, fresh ${ }^{4} \mathrm{He}$ which has been processed in the hydrogenburning shell accompanies the fresh ${ }^{12} \mathrm{C}$ outward to the surface.

Another important set of reactions which takes place in the convective shell is the chain ${ }^{14} N(\alpha, \gamma){ }^{18} F\left(e^{+}, \nu\right)^{18} O(\alpha, \gamma){ }^{22} N e$. The initial abundance of ${ }^{14} N$ is the CNO equilibrium value achieved in the hydrogen-burning shell and so is at a number abundance nearly equal to the total number abundance of the CNO elements in the matter out of which the star has been born. The conversion into ${ }^{22} \mathrm{Ne}$ is completed early on in the convective shell phase.

\section{3 $\mathrm{Can}^{13} \mathrm{C}$ or ${ }^{22} \mathrm{Ne}$ Act as a Neutron Source?}

The two major sources of neutrons for producing s-process isotopes are thought to be the reactions ${ }^{13} \mathrm{C}(\alpha, n){ }^{16} \mathrm{O}$ (Cameron 1955) and $\left.{ }^{22} \mathrm{Ne}(\alpha, n)\right)^{25} \mathrm{Mg}$ (Cameron 1961). The ${ }^{13} C$ source might be activated in an AGB star if the outer edge of the convective shell were to reach outward far enough to encompass the region where hydrogen-burning has not quite proceeded to completion. Hydrogen nuclei would be ingested by the convective shell, and as these nuclei made their way inward through the carbon-rich convective shell, they would eventually encounter sufficiently high temperatures that the ${ }^{12} C(p, \gamma)^{13} N$ reaction would occur, followed shortly by beta decay to ${ }^{13} C$. One might then imagine that the ${ }^{13} C$ nuclei would continue their way downward until encountering temperatures of the order of $150 \times 10^{6} \mathrm{~K}$, at which point the $(\alpha, n)$ reaction on ${ }^{13} C$ would liberate neutrons.

There are two difficulties with this scenario. The first is that, in all studies to date that include radiation pressure, the formal outer edge of the convective shell does not reach hydrogen-rich matter, although it comes tantalizingly close. A way of understanding this is that there exists an "entropy barrier" between the outer 
edge of the convective shell and the base of the hydrogen-rich region (Iben 1976). The maximum entropy achieved in the convective shell is always smaller than the entropy in the convective envelope, so that these two regions can never meet. The barrier is set up through the hydrogen abundance profile that defines the defunct hydrogen-burning shell.

The second difficulty is that, even if hydrogen were to be ingested by the convective shell, the ${ }^{13} C$ formed may not flow inward far enough to become a neutron source. That is, as hydrogen is convected downward to temperatures high enough to burn ${ }^{12} C$, the rate of entropy production by the ${ }^{12} C(\alpha, \gamma){ }^{13} N$ reaction may lead to the formation of a second convective shell which is sustained by hydrogen burning at its base and is separated by a radiative zone from the outer edge of the lower, helium-burning convective shell. Exactly this sort of detachment has been found in a low mass red giant model of initial population III composition when it ignites a helium shell flash very close to the hydrogen-burning shell (Hollowell, Iben, \& Fujimoto 1990). The outer edge of the helium-burning convective zone extends into hydrogen-rich material, and when the leading edge of the inwardly convected hydrogen profile reaches temperatures in the range $\sim 1-1.2 \times 10^{8} \mathrm{~K}$, the time scale for proton capture becomes shorter than the time scale for inward mixing. A new, hydrogen-burning convective layer forms and detaches from the heliumburning convective layer. The new convective shell grows outward as the overall rate of hydrogen burning outstrips the overall rate of helium burning. Thus, ${ }^{13} \mathrm{C}$ is formed, but subsequent reactions bring it into CNO cycle equilibrium abundance, and products of hydrogen burning fail to penetrate the radiative zone separating the two convective shells.

A more promising candidate for a neutron source in stars with massive cores may be the ${ }^{22} \mathrm{Ne}(\alpha, n)^{25} \mathrm{Mg}$ reaction. It is found that, for all $M_{C O} \gtrsim 1 M_{\odot}$, when the cross section for this reaction given by Fowler, Caughlan, \& Zimmerman (1975) is used, most of the ${ }^{22} \mathrm{Ne}$ formed early in the helium convective shell phase is converted into ${ }^{25} \mathrm{Mg}$, with the emission of a neutron (Iben 1977). For a typical population I composition, this translates into about 40 neutrons per ${ }^{56} \mathrm{Fe}$ seed nucleus. Unfortunately, recent experiments have shown that the uncertainty in the rate of the $(\alpha, n)$ reaction is so large that this result can not now be considered definitive (see Arnould 1991, this conference). The actual cross section may be such that the ${ }^{22} \mathrm{Ne}$ source is not activated in any AGB stars; it could equally well be large enough that ${ }^{22} \mathrm{Ne}$ acts as a strong neutron source also in AGB stars with core masses much smaller than $1 M_{\odot}$. Or, the standard rate may be correct (see $\$ 6.3$ below)! In any case, the results of Wood, Bessell, and Fox (1983) tell us that some neutron source is acting in real AGB stars with core masses larger than $1 M_{\odot}$, and ${ }^{22} \mathrm{Ne}$ remains the most likely source. 
5.4 Overlap of Convective Zones, the ${ }^{22} \mathrm{Ne}$ Neutron Source, and s-Process Nucleosynthesis

An important characteristic of successive convective shells is that they overlap in such a way that a substantial amount of matter that has been processed in any given convective shell is also processed in the following convective shell with the result that, in any given convective shell, the fraction of matter that has experienced $\mathbf{N}$ pulses of neutron irradiation is proportional to $e^{-\alpha N}$ where $\alpha$ is a constant which is determined by the degree of overlap (Ulrich 1973). The fraction of liberated neutrons that are captured by the seed nucleus ${ }^{56} \mathrm{Fe}$ and by its neutron rich progeny is determined by the number of light element filters with atomic numbers less than that of iron (Iben 1975b).

If the neutron source is ${ }^{22} \mathrm{Ne}$, the dominant filters are the ${ }^{25} \mathrm{Mg}$ that is made in the neutron-producing reaction along with the neutron rich progeny of ${ }^{25} \mathrm{Mg}$. The fact that, in this case, the number of filters is comparable with the number of released neutrons has as a consequence that, in broad outline, the final neutronrich distribution (after many pulses) is characterized primarily by the average cross section of the light element filters (Iben 1975b, Truran \& Iben 1977). The fact that this nearly unique distribution resembles in many respects the solar-system distribution of s-process isotopes was at one time thought to be a demonstration that AGB stars of large core mass are the major contributers of Galactic s-process isotopes and that ${ }^{22} \mathrm{Ne}$ is indeed the major neutron source.

However, at a number of critical branch points, the relative abundances of sprocess isotopes are very sensitive to the neutron density and to the temperature during successive neutron-nucleosynthesis episodes. It is now well established that, if the ${ }^{22} \mathrm{Ne}$ source is operating in AGB stars of large core mass, the neutron densities are so large that the isotopic abundances near several critical branch points are far from being in the solar system distribution (Despain 1980; Howard et al. 1986; Busso et al. 1988). Since the lifetime of such stars is of the order of ten times smaller than what it was thought to be when they were identified as the major source of s-process isotopes in the Galaxy (Iben \& Truran 1978), this does not necessarily mean that the ${ }^{22} \mathrm{Ne}$ source is not operating in their convective shells.

\subsection{The Dredge Up Mechanism}

During the helium-burning thermonuclear runaway, all of the energy produced by helium burning is confined to the convective shell (increasing the thermal energy and gravitational potential energy of matter in the shell). Only after the thermal instability has been quenched, and helium continues to burn in a quiescent fashion, does energy from helium-burning reactions leak into the hydrogen-rich envelope and make its way to the surface. At the same time, matter in the hydrogen-rich region is moving outward and cooling, with a consequent increase in the local radiative opacity. The net result is that the minimum condition for instability against con- 
vection, which involves a factor that is proportional to both the luminosity and the opacity, occurs at a point which moves progressively inward in mass; the base of the convective envelope therefore also moves inward in mass (but outward in radius!).

When no special care is exercised to take into account overshoot beyond the formal convective-radiative boundary and when "reasonable" values of $l / H$ are chosen, the base of the convective envelope moves into the region where some conversion of hydrogen into helium has occurred (Gingold 1974, 1975). If core mass is large enough $\left(M_{C O}>0.8-0.9 M_{\odot}\right)$, the base of the envelope convective zone moves further inward in mass into the top of the region once contained in a convective shell, where partial conversion of helium into carbon has occurred (Iben 1974, 1975a, 1976; Nomoto \& Sugimoto 1975; Fujimoto, Nomoto, \& Sugimoto 1975). Fresh carbon is mixed into the convective envelope, along with fresh s-process elements (presumably), and along with fresh helium (Renzini and Voli 1981).

\section{6 ${ }^{12} \mathrm{C},{ }^{7} \mathrm{Li}$, and Burning in the Convective Envelope}

Whether or not the ${ }^{12} C$ mixed into the envelope of an AGB model remains intact or whether it is burned into ${ }^{14} \mathrm{~N}$ at the base of the envelope depends on the choice of $l / H$. For example, in a model with a core of mass $M_{C O} \sim 0.96 M_{\odot},{ }^{12} C$ is converted into ${ }^{14} \mathrm{~N}$ on a time scale of only $\sim 20,000 \mathrm{yr}$ when $l / H \sim 1.0$ but does not burn at all if $l / H \sim 0.7$ (Iben 1975a). Thus, whether or not an AGB star with a large core mass becomes a carbon $\operatorname{star}\left({ }^{12} C>{ }^{16} O\right.$ by number) cannot be established from first principles.

The absence of carbon stars in the Magellanic clouds brighter than $M_{b o l} \sim$ 6 mag (Blanco, McCarthy, \& Blanco 1980) demonstrates that AGB stars with core masses larger than $\sim 0.8 M_{\odot}$ do not become carbon stars, but this could be due to the fact that such stars do not live long enough to dredge up sufficient carbon, even if the dredged-up carbon survives burning at the base of the convective envelope.

Several estimates of the lifetimes of AGB stars with large core masses can be made. In Magellanic cloud clusters for which ages and hence turnoff luminosities can be estimated (Frogel et al. 1990), one may combine observed properties with theory to estimate the initial core mass and hence the initial mean luminosity $L_{s t a r t}$ of TPAGB stars in the cluster. The luminosity $L_{\max }$ of the brightest TPAGB star in the cluster is known and is typically less than a factor of 2 larger than $L_{s t a r t}$. The theoretical rate at which the mean brightness of a TPAGB star increases is approximately $1 \mathrm{mag} / 10^{6} \mathrm{yr}$. From this and the knowlege of how many TPAGB stars there are with $L_{\text {star }}<L<L_{\max }$, one can estimate that the lifetime of AGB stars with massive cores is considerably smaller than $10^{6} \mathrm{yr}$.

Another way to estimate lifetimes is to compare the number of long period variables which are $\mathrm{ZrO}$-rich, TPAGB stars with large core masses (Wood et al. 1983) with the number of Cepheids. The ratio is less than 1 to 10 . Since the lifetime of a typical Cepheid. is $\sim 10^{6} y r$ (Becker, Iben, \& Tuggle 1977), the lifetime 
of TPAGB stars with massive cores is less than $\sim 10^{5} \mathrm{yr}$. Since it takes about $10^{6} \mathrm{yr}$ of dredging up for the amount of fresh carbon mixed into the convective envelope (plus the carbon already there at the outset of the TPAGB phase) to exceed the abundance of oxygen there (Iben \& Truran 1978, Iben 1981), real TPAGB stars cannot become carbon stars, whether or not carbon is converted into nitrogen at the base of the convective envelope.

The recent discovery that the ZrO-rich LPV's are also super lithium rich (Smith and Lambert 1989, 1990) will perhaps eventually allow an estimate of the temperature at the base of the convective envelopes of real AGB stars and thus settle the question as to what becomes of dredged-up carbon. It may also provide another constraint on the lifetime of luminous TPAGB stars. The origin of surface lithium is probably the mechanism invented by Cameron and Fowler (1971) and worked out in the context of AGB model envelopes by Sackmann, Smith, and Despain (1974). This mechanism assumes that some of the ${ }^{7} \mathrm{Be}$ which is made near the base of the convective envelope via the reaction ${ }^{3} \mathrm{He}(\alpha, \gamma){ }^{7} \mathrm{Be}$ can escape into the cool outer regions of the envelope before it decays into ${ }^{7} \mathrm{Li}$ and is destroyed by the ${ }^{7} \mathrm{Li}(\mathrm{p}, \alpha)^{4} \mathrm{He}$ reaction. The ${ }^{7} \mathrm{Be}$ beta decays into ${ }^{7} \mathrm{Li}$ as it is convected to the surface. The ${ }^{7} \mathrm{Li}$ is also convected inward to hot layers where it is destroyed, so some mean gradient in its abundance is established.

Sackmann et al. (1974) find that by (1)treating convective transport in a timedependent way, (2) adopting initial ${ }^{3} \mathrm{He}$ abundances given by evolutionary models beginning on the main sequence, (3) neglecting reactions which create ${ }^{3} \mathrm{He}$, and (4) adjusting the parameters of model convective envelopes optimally, this process can be made to yield peak surface $L i / H$ abundance ratios which are two or more orders of magnitude larger than solar. In their models, the $\mathrm{Li}$ abundance rises to a maximum, which is proportional to the initial abundance of ${ }^{3} \mathrm{He}$ and thereafter declines as the initial ${ }^{3} \mathrm{He}$ is used up. In a particular model designed to resemble an AGB star of mass $5 M_{\odot}$, the maximum value of the surface $L i / H$ ratio is $\sim$ 100 times solar, the time during which the surface $L i / H$ ratio remains larger than solar is $\sim 10^{5} \mathrm{yr}$ and the time during which it is ten times larger than solar is $\sim 3 \times 10^{4} \mathrm{yr}$.

The fact that all of the ZrO-rich LPV's which Smith and Lambert $(1989,1990)$ have observed thus far are super lithium rich might then be taken to indicate that their lifetimes as TPAGB stars are of the order of or less than a few times $10^{4} \mathrm{yr}$. However, the maximum surface abundance of $\mathrm{Li}$ achieved in the models is quite sensitive to the temperature assumed at the base of the convective envelope and the duration of the high lithium episode may be considerably longer when the reactions $p\left(p, e^{+} \nu\right) d(p, \gamma)^{3} \mathrm{He}$ which create ${ }^{3} \mathrm{He}$ are taken into account. That is, there may be a steady state surface $L i / H$ abundance ratio that is considerably larger than solar, in which case an argument regarding lifetimes cannot be made. It is clear that the ${ }^{7} \mathrm{Li}$ formation problem deserves further theoretical study. 
6. NUCLEOSYNTHESIS AND DREDGE UP IN AGB STARS WITH A SMALL CORE MASS

Since this topic is reviewed in a paper presented at this conference by Sackmann and Boothroyd (1991), the discussion here will be brief.

\subsection{Light Curves - Large Amplitude Variations and Minimum Core Mass for Dredge Up}

One of the important distinguishing features of TPAGB models of low mass is that the variation in surface luminosity is much more pronounced than in the case of large core masses. For example, at the depth of their light curve, models with $0.5<M_{C O} / M_{\odot}<0.6$ are about one magnitude dimmer than during the quiescent hydrogen-burning phase just prior to a thermal pulse. For AGB models with $M_{C O} \sim 1.0 M_{\odot}$ this difference is only about $0.5 \mathrm{mag}$. Further, for $30 \%$ of the time, they remain substantially dimmer than predicted by the famous Paczynski-Uus (P$\mathrm{U})$ core mass - luminosity relationship which is, stricly speaking, valid only at maximum luminosity just before the occurrence of a thermal pulse.

The variation in luminosity is important for an interpretation of the numbermagnitude distributions defined by carbon stars in clusters in both the Galaxy and the Magellanic clouds as well as for an interpretation of the distribution defined by field carbon stars in the Magellanic clouds. The dimmest carbon stars in the tails of each distribution are probably in the core helium-burning phase and the mass of the core estimated by using the P-U relationship for these dimmest stars is smaller than the actual core mass (Iben 1981, Iben \& Renzini 1983). The carbon-star number-magnitude distribution given by stars in the Bar West field of the LMC is a bell-shaped curve which extend from about $M_{b o l} \sim-4 \mathrm{mag}$ to $\sim-6 \mathrm{mag}$ (Blanco et al. 1980). Used blindly, the P-U relationship would predict a core mass of $\sim 0.55 M_{\odot}$ for the dimmest carbon stars. Assuming instead that they are actually one magnitude brighter at pre-pulse maximum, one obtains with the PU relationship that $M_{C O} \sim 0.63 M_{\odot}$ for the dimmest carbon stars. The actual core mass for the dimmest carbon stars is probably somewhere between these two values.

The time scales for variations in magnitude during a pulse and immediately thereafter are a function of the core mass. Hence, if magnitude determinations extending over a long enough period exist, observed variations can be used in a more direct fashion to estimate the core mass of an AGB star. In this way, Wood and Zarro (1981) have estimated the core mass of three nearby AGB stars. They estimate $0.61<M_{C O} / M_{\odot}<0.66$ for R Hya, which is an M star with a bolometric magnitude of $\sim-5 \mathrm{mag}$. The fact that $\mathrm{R} \mathrm{Hya}$ is not a carbon star while stars in the Magellanic clouds with similar core masses are suggests that, at any given core mass, the larger the metallicity, the more difficult it is to achieve a $\mathrm{C} / \mathrm{O}$ number ratio larger than unity. This is consistent with the fact that the frequency of carbon stars declines rapidly with increasing metallicity (Blanco, McCarthy, \& Blanco 1978). 
6.2 Semiconvection at the Edge of the Carbon-Rich Zone and Activation of the ${ }^{13} \mathrm{C}$ Neutron Source

As shell convection dies away in TPAGB models with a small core mass, matter that was once in the outer portions of the convective shell during pulse peak is propelled outward to much lower densities and temperatures than in the case of models with more massive cores. In particular, the temperature in matter at the edge of the carbon-rich zone drops to $\sim 10^{6} \mathrm{~K}$ or less. Sackmann (1981) points out that, at such low temperatures, carbon atoms will not be completely ionized and suggests that the opacity might be significantly enhanced over that obtained under the assumption of complete ionization and that this enhanced opacity might aid in the process of dredge up.

Using approximate fits to relevant opacity data in the literature, Iben and Renzini $(1982 \mathrm{a}, \mathrm{b})$ find that, in models of core mass $\sim 0.6 M_{\odot}$, a semiconvective region is set up at the outer edge of the carbon-rich layer constructed during the immediately preceding convective shell phase; within this semiconvective region, carbon is transported outward in such a way that the radiative temperature gradient exactly balances the adiabatic gradient. Some fresh carbon ultimately reaches the region where hydrogen is found and hydrogen is thereafter carried inward by semiconvective motions. At the end of this episode, there is left a narrow layer (of mass $\sim 4 \times 10^{-4} M_{\odot}$ ) in which carbon and hydrogen exist as trace elements at comparable number abundances. In the models of Hollowell (1988), the total mass of ${ }^{13} C$ in this layer is $\sim 5 \times 10^{-6} M_{\odot}$

Toward the end of the quiescent helium-burning phase, the matter in the ${ }^{13} C-{ }^{14} N$ layer has become hot and dense enough for the ${ }^{12} C(p, \gamma){ }^{13} N\left(e^{+}, \nu\right)^{13} C$ and the ${ }^{13} C(p, \gamma)^{14} N$ reactions to operate. Over the inner portion of the layer, where there is initially less than one proton per ${ }^{12} C$ nucleus, the major product is ${ }^{13} \mathrm{C}$. Over the outer portions of the layer, where there is initially more than one proton per ${ }^{12} \mathrm{C}$ nucleus, the major product is ${ }^{14} \mathrm{~N}$.

Quiescent hydrogen burning then creates a blanket of ${ }^{4} \mathrm{He}$ and ${ }^{14} \mathrm{~N}$ above the ${ }^{13} \mathrm{C}-{ }^{14} \mathrm{~N}$ layer and, at the start of the next thermal pulse, the ${ }^{13} \mathrm{C}-{ }^{14} \mathrm{~N}$ layer finds itself approximately midway (in mass) between the base of the carbon- and helium-rich layer and the base of the hydrogen-burning shell. When the outer edge of the convective shell formed during this next pulse encounters the ${ }^{13} C$-rich layer, ${ }^{13} \mathrm{C}$ is introduced into the convective shell over a period of approximately 3-10 yr. At the time of introduction, the temperature at the base of the convective shell is $\sim 150 \times 10^{6} \mathrm{~K}$, so that the ${ }^{13} \mathrm{C}$ which is convected inward becomes a source of neutrons near the base of the convective shell.

After all of the ingested ${ }^{13} \mathrm{C}$ has been destroyed, the convective shell continues to grow in mass and to incorporate more of the ${ }^{4} \mathrm{He}$ and ${ }^{14} \mathrm{~N}$ which has been processed by the hydrogen-burning shell during the preceding hydrogen-burning phase. The ingested ${ }^{14} \mathrm{~N}$ is converted into ${ }^{22} \mathrm{Ne}$ as the temperature at the base of 
the convective shell continues to rise toward a maximum of $\sim 300 \times 10^{6} \mathrm{~K}$. During a brief period when temperatures are near this maximum, approximately $1 \%$ of the ${ }^{22} \mathrm{Ne}$ originating from ${ }^{14} \mathrm{~N}$ is converted into ${ }^{25} \mathrm{Mg}$ with the release of a neutron (Becker 1981, Iben 1982). The figure of $1 \%$ is based on the assumption that the cross section given by Fowler et al. (1975) is correct.

\subsection{Production of s-Process Isotopes in the Solar-System Distribution}

During the ${ }^{13} C$ ingestion phase, the timescale for the release of neutrons at the base of the convective shell is set by the rate at which ${ }^{13} C$ is ingested and this timescale is two orders of magnitude longer than the timescale of the ${ }^{13} C(\alpha, n){ }^{16} O$ reaction (Iben 1983). It is this characteristic that leads to neutron densities of the correct order of magnitude for the production of s-process isotopes in the solar system distribution (Gallino et al. 1988; Gallino 1989; Hollowell 1988; Hollowell \& Iben 1988, 1989, 1990).

A typical turnover time for matter in the convective shell is about half a day and a typical ${ }^{13} \mathrm{C}$ nucleus cycles back and forth in the shell approximately 30 times before it captures an alpha particle near the base of the shell. The time dependence of the neutron density in the region where most of the neutron capture nucleosynthesis occurs is of interest. In their pulse model based on an AGB model by Iben (1982), Gallino et al. (1988) find that this density rises rapidly to $\sim 10^{9} \mathrm{~cm}^{-3}$ and then rises more slowly to a maximum of $\sim 4 \times 10^{9} \mathrm{~cm}^{-3}$ at the end of the ingestion period. After this, neutrons continue to be released at a steadily declining rate over a time scale that depends on the choice of the uncertain cross section for the ${ }^{12} C(n, \gamma){ }^{13} C$ cross section. Hollowell and Iben (1990) show that the history of the relevant neutron density can be obtained from a one zone model using input data from their AGB models, and find neutron-density histories very much like those found by Gallino et al. (1988) and by Gallino (1989).

With very comprehensive reaction networks, Gallino and his collaborators (Gallino et al. 1988, Gallino 1989, Käppeler et al. 1990, Picchio et al. 1990) have found excellent agreement (within the uncertainties) between the distribution of neutron-rich isotopes calculated in the context of detailed AGB models and the solar system distribution of s-process isotopes. In particular, solar system abundance ratios are found for isotopes in the neighborhood of branch points where abundance ratios are separately sensitive to the neutron density, to the temperature, and to the matter density (Käppeler et al. 1990).

A particularly interesting result is that the weak neutron irradiation which occurs after the main neutron irradiation episode when the temperature at the base of the convective shell reaches $\sim 300 \times 10^{6} \mathrm{~K}$ and the ${ }^{22} \mathrm{Ne}$ source is activated appears to be essential for obtaining the solar system abundance distribution near three temperature-sensitive branch points involving, respectively, ${ }^{152} G d,{ }^{164} \mathrm{Er}$, and ${ }^{180} \mathrm{Ta}$ (Käppeler et al. 1990). These results may prove to be a very strong constraint 
on the experimental estimates of the cross section for the ${ }^{22} \mathrm{Ne}(\alpha, n)^{25} \mathrm{Mg}$ reaction at energies of $\sim 1 \mathrm{MeV}$.

The major weakness in the theory presented here rests with the AGB models or possibly with the opacity used to construct these models. Thus far, the ${ }^{13} C$ neutron source has been found to be activated only in low metallicity models, whereas it is known that population I stars produce neutron-rich isotopes in approximately the solar system s-process distribution (Lambert 1989, 1991). In order to achieve semiconvection at the edge of the carbon-rich zone, the contribution of carbon to opacity must be significant relative to the contribution of other elements and the larger the metallicity, the smaller this relative contribution will be. Is it possible that a reevaluation of opacities for carbon-rich, helium-rich, and metal-rich matter at low temperatures is in order?

\section{4 "Extramixing", $l / H$, and Dredge Up}

Prior to the work of Blanco et al. (1980), dredge up was not encountered in TPAGB models constructed with radiation pressure taken into account and with core masses as small as $0.6 M_{\odot}$. When convective overshoot beyond the base of the convective envelope is taken into account, dredge up does occur in such models (Iben and Renzini 1982a,b; Iben 1983).

Hollowell (1988), using a much better approximation to the opacity in the carbon-rich region and a time-independent treatment of convective mixing, demonstrates that, without including overshoot, dredge up does not occur, despite the increase in opacity in the semiconvective layers which are formed. Using a timedependent algorithm for convective mixing which naturally estimates the degree of overshoot, he also finds mixing.

The Iben-Renzini work and the Hollowell work assume "reasonable" values for $l / H$. Dredge up can also be achieved in models of small core mass by choosing much larger values of this parameter, as shown by Boothroyd and Sackman (1988a,b) and by Lattanzio (1989 and references therein). Given the crudeness of the mixing length treatment of convection, who is to say that such large values are inappropriate?

\section{Acknowledgements}

This work has been supported in part by the USA National Science Foundation grant AST88-00773, discretionary funds associated with the Eberly Family Chair in Astronomy and Astrophysics at the Pennsylvania State University, a Japan Society for the Promotion of Science Fellowship, and a Grant-in-Aid for Scientific Research of the Ministry of Education, Science, and Culture (63540191) at the University of Niigata. It is a pleasure to thank Masayuki Fujimoto and the University of Niigata for providing an environment conducive to the preparation of this contribution. 


\section{REFERENCES}

Arnould, M. 1991, in Evolution of Stars: The Photospheric Abundance Connection, ed. G. Michaud and A. V. Tutukov 1991 (Dordrecht: Kluwer), p. $\mathbf{x x}$

Becker, S. A. 1981, in Physical Processes in Red Giants, ed. I. Iben, Jr. and A. Renzini (Dordrecht: Reidel), p. 141

Becker, S. A., Iben, I. Jr., and Tuggle, R. S. 1977, ApJ, 218, 633

van den Bergh, S., McClure, R. D., and Evans, R. 1987, ApJ, 323, 44

Blanco, B. M., McCarthy, M. F., and Blanco, V. M. 1978, Nature, 271, 638

Blanco, V. M., McCarthy, M. F., and Blanco, B. M. 1980, ApJ, 242

Blöcker, T., and Schönberner, D. 1990, A\&A, in press

Boothroyd, A. I., and Sackman, I.-J. 1988a, ApJ, 328, 653 1988b, ApJ, 328, 671

Bowen, G. H. 1989, in Evolution of Peculiar Red Giants, ed. H. R. Johnson and B. Zuckerman (Cambridge: Cambridge U.), p. 284.

Busso, M., Picchio, G., Gallino, R., and Chieffi, A. 1988, ApJ, 326, 196

Cameron, A. G. W. 1955, Ap. J., 121, 144 1961, AJ, 65, 485 1973, in Explosive Nucleosynthesis, ed. D. W. Schramm and D. W. Arnett (Austin: U. Texas), p. 3.

Cameron, A. G. W., and Fowler, W. F. 1971, ApJ, 164, 111

Despain, K. H. 1980, ApJL, 236, L165

Evans, R., van den Bergh, S., and McClure, R. D. 1989, ApJ, 345, 752

Frogel, J. A., Mould, J., and Blanco, V. M. 1990, ApJ, 352, 96

Gingold, R. 1974, ApJ, 193, 177 1975, ApJ,198, 425

Fowler, W. F., Caughlan, G. R., and Zimmerman, B. A. 1975, ARAAp, 13, 69

Fujimoto, M. Y., and Iben, I. Jr. 1990, APJ, in press

Fujimoto, M. Y., Nomoto, K., and Sugimoto, D. 1975, PASJ, 28, 89

Gallino, R. 1989, in Evolution of Peculiar Red Giants, ed. H. R. Johnson and B. Zuckerman (Cambridge: Cambridge U.), p. 176.

Gallino, R., Busso, M., Picchio, G., Raiteri, C. M., and Renzini, A. 1988, ApJL, 334, L45

Hollowell, D. 1988, Ph.D. Thesis, University of Illinois

Hollowell, D., and Iben, I. Jr. 1988, ApJL, 333, L25.

1989, ApJ, 340, 966 1990, ApJ, 349, 208

Hollowell, D., Iben, I. Jr., and Fujimoto, M. Y. 1990, ApJ, in press 
Howard, W. M., Mathews, G. J., Takahashi, K., and Ward, R. A. 1986, ApJ, 309,633

Iben, I. Jr. 1974 ,BAAS, 6, 316

1975a, ApJ, 196, 525

1975b, ApJ, 196, 549

1976, ApJ, 208, 165

1977, ApJ, 217, 788

1981, ApJ, 246, 278

1982, ApJ, 260, 821

1983, ApJL, 275, L65

Iben, I. Jr., and Renzini, A. 1982a, ApJL, 259, L79

1982b, ApJL, 263, L23

1983, ARA\&A, 21, 271

Iben, I. Jr., and Truran, J. W. 1978, ApJ, 220, 980

Iben, I. Jr., and Tutukov, A. V. 1989, ApJ, 342, 430.

Jura, M. 1989, in Evolution of Peculiar Red Giants, ed. H. R. Johnson and

B. Zuckerman (Cambridge: Cambridge U.), p. 337.

Knapp, G. R., and Morris, M. 1985, ApJ, 292, 640

Käppeler, F., Gallino, R., Busso, M., Picchio, G., and Raiteri, C. M. 1990, ApJ, 354, 630.

Lambert, D. L. 1989, in Evolution of Peculiar Red Giants, ed. H. R. Johnson and B. Zuckerman (Cambridge: Cambridge U.), p. 101.

1991, in Evolution of Stars: The Photospheric Abundance

Connection, ed. G. Michaud and A. V. Tutukov 1991 (Dordrecht: Kluwer), p. $\mathbf{x x}$

Little-Marenin, I. R. 1989, in Evolution of Peculiar Red Giants, ed. H. R.

Johnson and B. Zuckerman (Cambridge: Cambridge U.), p. 101.

Lattanzio, J. 1986, ApJ, 311, 708

1987, ApJL, 313, L15

1989, in Evolution of Peculiar Red Giants, ed. H. R. Johnson and

B. Zuckerman (Cambridge: Cambridge U.), p. 161.

Miyaji, S., and Nomoto, K. 1987, ApJ, 318, 307

Nomoto, K. 1984, ApJ, 277, 791 1987, ApJ, 322, 206

Nomoto, K. , and Sugimoto, D. 1975, PASJ, 27, 197

Picchio, G., Busso, M., Gallino, R., and Raiteri, C. M. 1990, in preparation

Reimers, 1978, Mem. Soc. Roy. Sci. Liege, 6 e Ser. 8, 369

Renzini, A., and Voli, M. 1981, A\&A, 94, 175

Sackmann, I.-J. 1980, ApJL, 241, L37 
Sackmann, I.-J., and Boothroyd, A. I. 1991, in Evolution of Stars: The Photospheric Abundance Connection, ed. G. Michaud and A. V. Tutukov 1991 (Dordrecht: Kluwer), p. xx

Sackman, I.-J., Smith, R. L., and Despain, K. H. 1974, ApJ, 187, 555.

Smith, V. V., and Lambert, D. L. 1989, ApJL, 345, L75 1990 , ApJL, in press.

Truran, J. W., and Iben, I. Jr. 1977, ApJ, 216, 797

Ulrich, R. K. 1973, in Explosive Nucleosynthesis, ed. D. N. Schramm and D. W. Arnett (Austin: U. Texas), p. 139

Wood, P. R. 1981, in Physical Processes in Red Giants, ed. I. Iben, Jr. and A. Renzini (Dordrecht: Reidel), p. 205

Wood, P. R., Bessell, M. S., and Fox, M. W. 1983, ApJ, 272, 99

Wood, P. R., and Zarro, D. 1981, ApJ, 247, 247 